\title{
CT PULMONARY ANGIOGRAM WITH REDUCED RADIATION EXPOSURE AT LOW TUBE KILOVOLTAGE
}

\author{
Ahmed A. Hashi ${ }^{1}$ \\ ${ }^{1}$ PhD Scholar, Medical School in Radiology, Australian National University \\ *Correspondence email: ahmed.hashi@anu.edu.au
}

\begin{abstract}
This study's primary goal is to assess the image quality and radiation dose of the low-dose $80 \mathrm{kV}$ computed tomography pulmonary angiogram (CTPA) protocol compared to the standard 100kV CTPA protocol for the assessment of pulmonary embolism (PE). The study consisted of 100 patients who had clinically suspected pulmonary embolism and required a CTPA. Patients underwent imaging with a 320-row multi-detector Toshiba Aquilion One Genesis Edition in the absence of the proprietary radiation reduction software known as forward projected model-based Iterative Reconstruction Solution (commercial acronym 'FIRST'). Participants were divided into two groups: A and B. Group A was composed of 50 patients allocated to standard CT protocol using a $100 \mathrm{kV}$ exposure setting and all other settings set as a standard by the manufacturer. Group B was composed of 50 patients who were allocated to a CTPA with a low-dose $80 \mathrm{kV}$ protocol, standard deviation level 8 , an effective mAs of 258, reconstruction algorithm-kernel FC 51 within the lung window, and tube current modulation. A considerable decrease in radiation dose was observed with the low-dose CTPA protocol. The mean radiation dose was also decreased by $66 \%$ while using the $80 \mathrm{kV}$ protocol than when utilizing a standard $100 \mathrm{kV}$ technique; this was achieved without compromising this study's diagnostic value. Furthermore, the contrast enhancement was considerably more significant, up to $40 \%$ higher when using $80 \mathrm{kV}$. The study found that a low tube voltage of $80 \mathrm{kV}$ CTPA protocol resulted in a considerable decrease in radiation dose and improved contrast enhancement without sacrificing the examinations' diagnostic utility.
\end{abstract}

Keywords: Low do and image quality of $80 \mathrm{kV}$; CT pulmonary angiogram; Low tube voltage; 80kV CTPA protocol; $100 \mathrm{kV}$ versus $80 \mathrm{kV}$, Image quality and contrast enhancement assessment of $80 \mathrm{~V}$.

\section{INTRODUCTION}

Pulmonary embolism (PE) is a possibly fatal disorder with persistent poor outcome among hospitalized patients ${ }^{1}$. Most PEs emerge due to deep vein thrombus (DVT or blood clots) in the extremities, most often the legs and pelvis. The moment any thrombus is created, it may extricate, move to the inferior vena cava, eventually passing via the right ventricle in the pulmonary vasculature $^{2}$. While most emboli are small and can be asymptomatic; occasionally, there are massive emboli which can cause symptoms and may lead to death in $30 \%$ of the instances by damaging the right ventricular output ${ }^{3,4}$. Thus patients with typical symptoms from PE should undergo timely diagnosis and urgent commencement of appropriate treatment. ${ }^{5}$

CT pulmonary angiogram (CTPA) is often utilized, and it is an ideal imaging technique used for diagnosing PE. CT imaging, in most cases, has considerable advantages over other types of imaging modalities. For example, it has much more widespread availability, availability after hours, and fast image acquisition in the emergency department with little preparation required, and it also has high diagnostic accuracy. Besides PE, it can also show other diseases where pulmonary embolism is not the source of the symptoms, such as pneumonia or dissection. CTPA is also easy for physicians to interpret images once images are reconstructed. Such merits influence physicians to over-use CTPA, leading to $89 \%$ of surveys being negative. Over-ordering CTPA with high radiation doses raises concerns about increased radiation exposure to patients. It is recognized that radiation exposure is linked to the possibility of developing breast cancer, particularly among pregnant and young patients. Therefore, appropriate radiation dose reduction techniques are required in the absence of damaging the quality of the images, as a significant drop in radiation dose may result in diminished image quality and consequently missed PE and alternative diagnoses.

This study's main purpose is to present a novel low-dose CTPA protocol to ensure that 
radiation exposure is as low as realistically possible in the absence of affecting the image quality and diagnostic utility. Reducing radiation is possible by utilizing different dose reduction methods, for example, altering the reconstruction algorithm-kernel, adjusting the standard deviation (SD), and utilizing low tube voltage $(80 \mathrm{kV})$ with tube current modulation, and changing the image reconstruction process to improve image quality.

\section{MATERIAL AND METHODS}

The study involved 100 patients with suspected PE who required CTPA. Patients underwent imaging on a Toshiba 320-row multidetector in the absence of the software for radiation reduction know as FIRST (commercial acronym). The study participants were categorized into two groups: Group A with standard CTPA protocol (control) and group B with the new low dose CTPA protocol (test). Each of the control and test groups consisted of 25 women and 25 men patients. To ensure consistency, pair matching was conducted based on similar age and weight distribution, as these are the most critical factors to control for radiation dose. Given ensuring similarity between the groups, the mean age of the participants in the control group, A was 56.050 \pm 19.66 years, whereas, for the test group B, it was $54.06 \pm 21.52$. The mean weight of the participants in control group A was $69.88 \pm 14.23 \mathrm{~kg}$, whereas, for test group B, it was $68.96 \pm 13.45 \mathrm{~kg}$.

Group A included 50 patients allocated to the standard CTPA $100 \mathrm{kV}$ procedure with reconstruction algorithm-kernel FC 53 with tube current modulation, the image reconstruction process AID 3D standard, and an effective mAs of 215 . This data was gathered before implementing a low dose CT pulmonary angiogram protocol. Group B was allocated to low-dose CTPA with the image reconstruction process AID 3D strong, standard deviation setting of level 8 (Sure Exposure 3D), an effective mAs of 258, and $80 \mathrm{kV}$ as well as the reconstruction algorithm-kernel FC 51 in the lung window incorporated with tube current modulation.

All the imaging was obtained in a sole breath-hold and craniocaudal manner. The injection rate was similar between the patients; $40-70 \mathrm{~mL}$ iodinated contrast medium (iopromide, commercial name Ultravist $^{\circledR}$ Bayer pharmaceuticals) was administered with a $50 \mathrm{~mL}$ saline flush. A minimum 18-G cannula within the cubital fossa was utilized with a $4.5 \mathrm{~mL} / \mathrm{sec}$ flow rate through a dual-headed injector. An automated bolus tracking system was formulated with a scanning trigger at $180 \mathrm{HU}$ and region of interest (ROI) positioned within the pulmonary trunk. ROI size was set at five $\mathrm{mm}^{2}$. Two experienced radiologists with over eight years of experience reported the studies. The image quality of both groups was evaluated using a 3-point scale. For example, score 1: Images with no diagnostic issue and/or minimal noise (excellent image quality). Score 2: Images with no diagnostic problem but with minor increased image noise (good image quality). Score 3: Images with noticeable image quality issues and/or significant image noise (suboptimal image quality). In the case of disagreeing scores in the study group's subjective image analysis, where one radiologist said suboptimal, and the other disagreed, images were reanalyzed, and consensus between the two radiologists was reached.

The study excluded patients under 18 years, patients suffering from kidney failure with an estimated Glomerular Filtration Rate $(e G F R<30)$ and chest depth greater than $30 \mathrm{~cm}$ or weigh over $105 \mathrm{~kg}$. To evaluate contrast enhancement, specifically to achieve the correct measurement in Hounsfield units (HU), a region of interest was positioned at the pulmonary trunk. Images that demonstrated contrast enhancement more than $210 \mathrm{HU}$ were accepted for having satisfactory contrast enhancement to detect $\mathrm{PE}^{6}$. The images were then ranked as suboptimal or non-diagnostic in cases where the contrast enhancement was lower than $210 \mathrm{HU}$ or if the reporting radiologist graded the images as non-diagnostic or suboptimal. The radiologist provided the final assessment of imaging or diagnostic quality.

The data were presented in terms of statistical properties, such as minimum, mean, and maximum (with confidence interval) of radiation doses and contrast enhancement. The study presented the frequency distribution of the list of PE alternative or differential diagnosis.

The outcome variables, radiation dose, and contrast enhancement were measured using standard techniques. Side by side box plots was presented to visualize the differences and to show the distribution of the radiation dose and contrast enhancement

A hypothesis test was conducted to test if significant differences exist between the mean of radiation dose $100 \mathrm{kV}$ protocol and $80 \mathrm{kV}$ protocol. For this purpose test, independent samples $t$-test with unequal variance were utilized to compare the radiation doses of the $80 \mathrm{kV}$ protocol and standard protocol. Radiologists' findings on 
diagnostic confidence and image quality were also presented to confirm or reject the hypothesis.

\section{RESULT}

The study involved 100 patients who were clinically thought to have PE and were recommended to the imaging department to rule out pulmonary embolism. A total of 15 positive instances for pulmonary embolism were identified. Among this group, $10 \mathrm{PE}$ diagnoses were identified within the standard CT protocol (control group), and 5 cases of PE were diagnosed in the low-dose CTPA group (test group). Alternative diagnoses, including pneumonia and emphysema, were also made in both groups; these are present in Tables 1 and 2.

In terms of radiation dose, a considerable decrease of approximately $66 \%$ in the effective dose was identified while utilizing an $80 \mathrm{kV}$ protocol compared to the control group. The $80 \mathrm{kV}$ protocol had an average effective dose that was significantly lower $(1.005 \mathrm{mSv})$ compared with the standard $100 \mathrm{kV}$ protocol $(3.03 \mathrm{mSv})$, as demonstrated in figure1, $(\mathrm{P}<0.05)$. This will be further discussed in the following section.

The study also found a significant improvement in control enhancement between the two groups. The average contrast enhancement in the pulmonary trunk was 643 in the low-dose protocol compared to 387 in the standard or control CTPA protocol; this is present in figure 2 . The contrast enhancement was increased by $60 \%$ with the low dose protocol $(\mathrm{p}<0.05)$.

There was a substantial difference between the groups. The control group and test groups were similar in quality and suggesting similar in diagnostic utility. This will also be discussed later.

Table 1 Alternative diagnoses with the standard dose CT pulmonary angiogram

\begin{tabular}{|c|c|}
\hline \multicolumn{2}{|c|}{ Radiologists' findings on standard CT pulmonary angiogram } \\
\hline Radiologists' findings & No. \\
\hline Normal studies & 24 \\
\hline $\mathrm{PE}$ & 10 \\
\hline Consolidation/infections & 4 \\
\hline Lung cancer/metastasis & 4 \\
\hline Atelectasis & 2 \\
\hline Lung nodules & 2 \\
\hline Emphysema & 1 \\
\hline Pleural effusion & 1 \\
\hline Lymphadenopathy & 1 \\
\hline Pulmonary edema & 1 \\
\hline
\end{tabular}

Table 2 Alternative diagnoses with the low-dose CT pulmonary angiogram

\begin{tabular}{|l|l|}
\hline Radiologists' findings on low-dose CT pulmonary angiogram & \\
\hline Radiologists' findings & No. \\
\hline Normal studies & 24 \\
\hline PE & 5 \\
\hline Emphysema & 4 \\
\hline Lung cancer/metastasis & 4 \\
\hline Atelectasis & 3 \\
\hline Lung nodules & 3 \\
\hline Consolidation/infections & 3 \\
\hline Pleural effusion & 2 \\
\hline Pulmonary edema & 2 \\
\hline
\end{tabular}




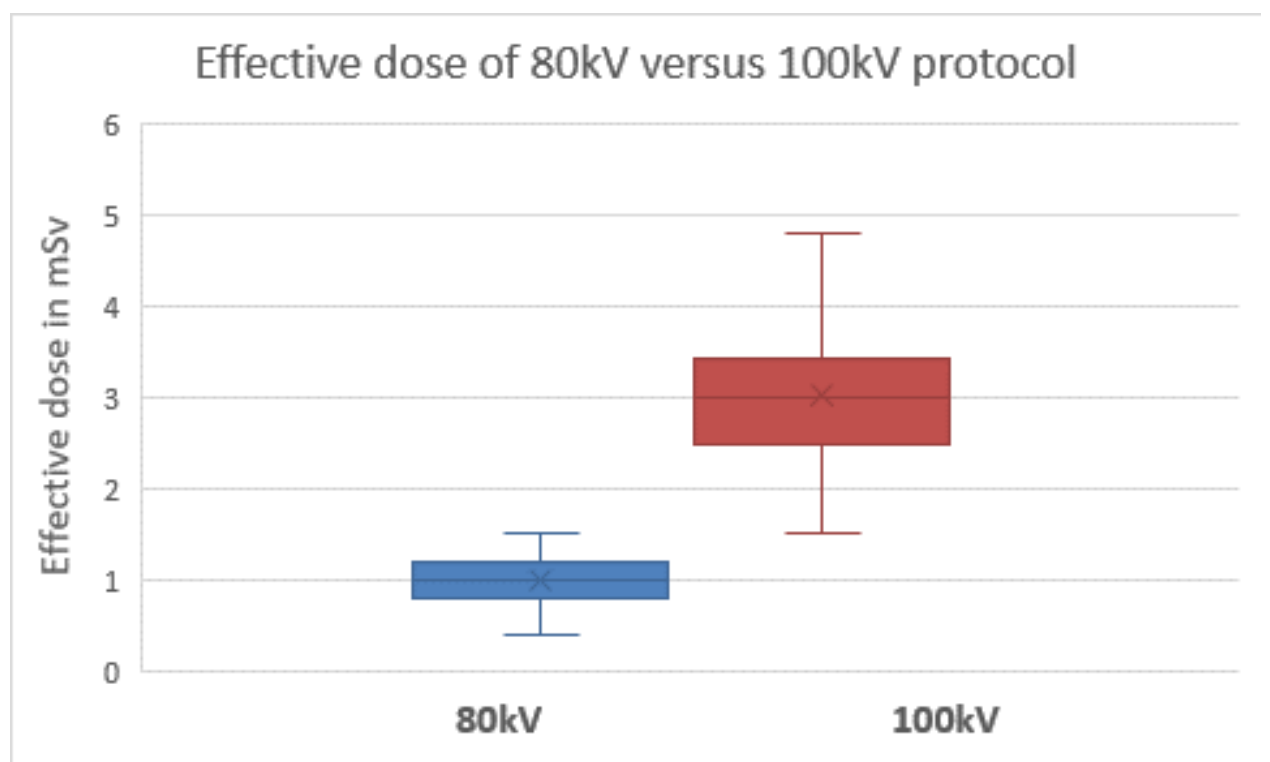

Figure 1. Box-whisker plot chart shows the distribution of radiation dose $100 \mathrm{kV}$ versus $80 \mathrm{kV}$.

\section{Radiation Dose Statistical Analysis}

The results for both the control and test protocols show that the data is approximately normally distributed. The standard CTPA $100 \mathrm{kV}$ protocol had a relatively higher effective dose than the $80 \mathrm{kV}$ protocol. The maximum and minimum exposure with the control $100 \mathrm{kV}$ protocol was $4.8 \mathrm{mSv}$ and $1.5 \mathrm{mSv}$, respectively, with a mean $3.03 \mathrm{mSv}$. With the $80 \mathrm{kV}$ protocol, the maximum and minimum exposure were $1.5 \mathrm{mSv}$, and $0.41 \mathrm{mSv}$, respectively, with a mean $1.005 \mathrm{mSv}$. The low dose $80 \mathrm{kV}$ protocol also had a relatively smaller variation than the $100 \mathrm{kV}$ protocol concerning the interquartile range (IQR). Table 3and 4 represents the results from a descriptive data analysis of radiation dose for $100 \mathrm{kv}$ and $80 \mathrm{kv}$ protocols.

The approximately normal distribution of both data sets and appropriately sufficient sample sizes allow us to utilize the independent samples $t$-test with an unequal variance to compare the protocols' mean radiation doses. This demonstrated that there existed a statistically significant difference $(\mathrm{t}(60)=-17.8, \mathrm{p}<0.05)$ in the radiation doses between the $80 \mathrm{kV}$ and $100 \mathrm{kV}$ protocols.

If the null hypothesis is set as the radiation exposure at $80 \mathrm{kV}$ and $100 \mathrm{kV}$ were equal, the alternative hypothesis was set as the radiation exposure at $80 \mathrm{kV}$ was less than $100 \mathrm{kV}$. The alternative hypothesis can be accepted.

Table 3 shows the statistical $\mathrm{t}$-Testing

\begin{tabular}{|l|l|l|}
\hline \multicolumn{2}{|l|}{ t-Test: Two-Sample Assuming Unequal Variances } \\
\hline & $K V 80$, dose $(\mathrm{mSv})$ & $100 \mathrm{kv}$ Dose in $\mathrm{mSv}$ \\
\hline Mean & 1.0054 & 3.03 \\
\hline Variance & 0.066547796 & 0.577653061 \\
\hline Observations & 50 & 50 \\
\hline Hypothesized Mean Difference & 0 & \\
\hline df & 60 & \\
\hline t Stat & -17.83666212 & \\
\hline $\mathrm{P}(\mathrm{T}<=\mathrm{t})$ one-tail & $5.76719 \mathrm{E}-26$ & \\
\hline t Critical one-tail & 1.670648865 & \\
\hline $\mathrm{P}(\mathrm{T}<=\mathrm{t})$ two-tail & $1.15344 \mathrm{E}-25$ & \\
\hline t Critical two-tail & 2.000297822 & \\
\hline
\end{tabular}


Table 4 shows a statistically significant difference

\begin{tabular}{|l|l|l|l|l|}
\hline Dose & Mean & SD & $t(d f)$ & $p$ \\
\hline $100 \mathrm{kV}$ & 3.03 & 0.577653061 & $1.15(60)$ & $5.76719 \times 10^{-26}$ \\
\cline { 1 - 3 } $80 \mathrm{kV}$ & 1.1 & 0.066547796 & & \\
\hline
\end{tabular}

$$
\begin{gathered}
\mathrm{H}_{0}: \mu_{\text {Dose } 80 \mathrm{kv}}=\mu_{\text {Dose } 100 \mathrm{kv}} \\
\mathrm{H}_{\mathrm{A}}: \mu_{\text {Dose } 80 \mathrm{kv}}<\mu_{\text {Dose } 100 \mathrm{kv}}
\end{gathered}
$$

$H_{a}$ : The alternative hypothesis validates that radiation exposure from low dose CT pulmonary angiogram is less than the radiation dose from $100 \mathrm{kV}$ protocol.

\section{Image Quality and Contrast Enhancement Assessment}

The maximum and minimum contrast enhancement with the $100 \mathrm{kV}$ protocol (control group) was $641 \mathrm{HU}$ and $153 \mathrm{HU}$, respectively, with a mean of $387 \mathrm{HU}$. On the other hand, with the $80 \mathrm{kV}$ protocol (test group), the maximum and minimum contrast enhancement were $1070 \mathrm{HU}$ and $337 \mathrm{HU}$, respectively, with a mean of 643HU (Table 5). The $100 \mathrm{kV}$ has a relatively smaller variation than the $80 \mathrm{kV}$ protocol with respect to the interquartile range (IQR). If the null hypothesis is set as the contrast enhancement at $80 \mathrm{kV}$ and $100 \mathrm{kV}$ were equal, and the alternative hypothesis was set as the contrast enhancement at $80 \mathrm{kV}$ was more than $100 \mathrm{kV}$, then the alternative hypothesis can be accepted.

Regarding imaging quality assessment, the low dose CTPA protocol yielded acceptable image quality comparable to the standard protocol as per the radiologist assessment. With the low dose protocol, 2 cases had a suboptimal or slightly optimal imaging quality, a single patient with chronic cardiac failure demonstrated reduced opacification of contrast, and another single patient had marked respiratory motion artifact. With the standard $100 \mathrm{kV}$ protocol, six examinations had suboptimal imaging quality. These were due to respiratory motion artifact and low contrast enhancement (Table 6). Therefore radiologists showed comparable confidence in detecting PE between low dose and standard CTPA protocols; this is bourne out in hypothesis testing. If the null hypothesis is set as the diagnostic confidence at $80 \mathrm{kV}$ was not equal to $100 \mathrm{k}$, and the alternative hypothesis was set as the diagnostic confidence was equal, the p-value was $>0.05$, and the alternative hypothesis was accepted, indicating being similar between protocols. Radiologist's image evaluation, as well as Chi-square test, show that the quality of CT pulmonary angiogram in the low dose and standard $100 \mathrm{kV}$ protocol is similar in image quality to diagnose or exclude pulmonary embolism (Figure 3 and 4).

The study also discovered a minor increase in image noise with the low dose protocol; this was noticeable on lung windows. The 9 cases identified to have minor image noise did not hamper the study's radiologists' diagnostic confidence. Overall, the radiologists indicated no difference in diagnostic accuracy and image quality with the low-dose protocol compared to the standard protocol. The radiologist also found no difference in confidence for diagnosing alternative diagnoses such as lung atelectasis, emphysema, large nodules, masses, and pneumonia. 


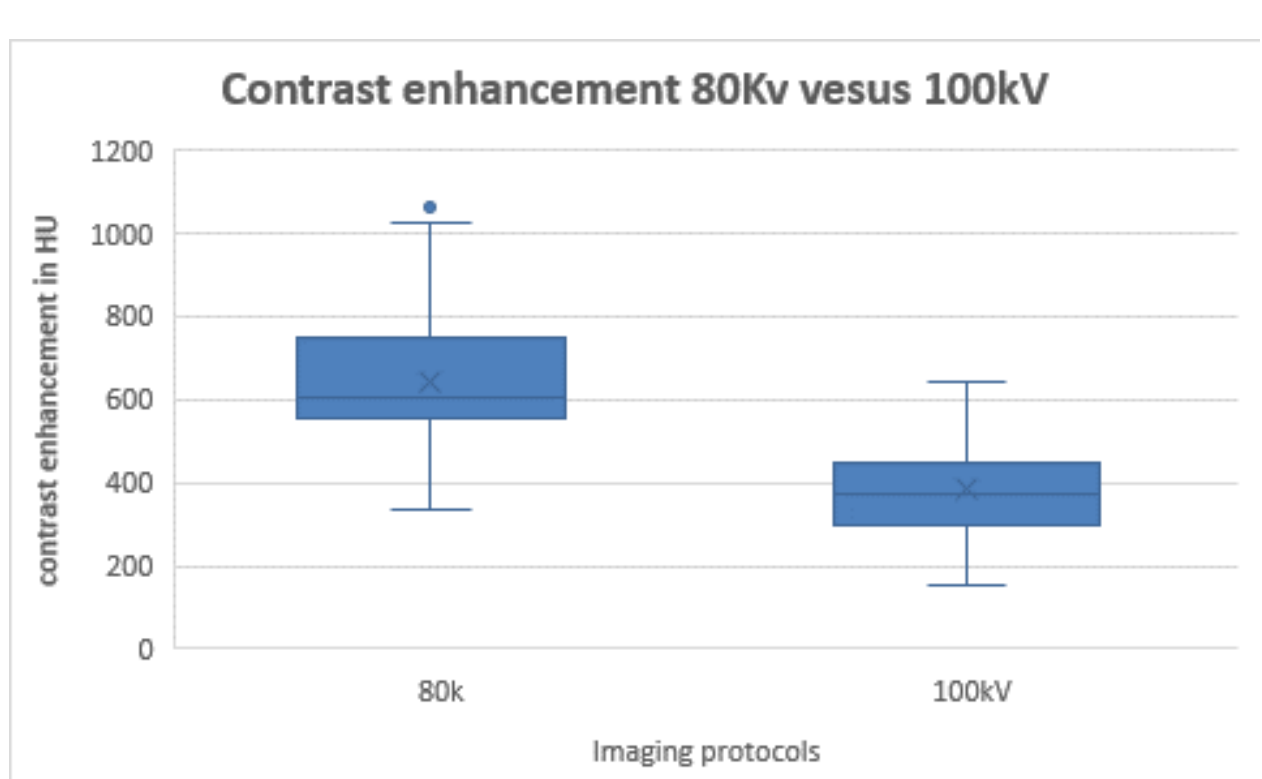

Figure 2 Box-whisker plot chart shows contrast enhancement $100 \mathrm{kV}$ versus $80 \mathrm{kV}$.

Table 5 shows statistical $\mathrm{t}$-Testing of contrast enhancement

\begin{tabular}{|l|l|l|}
\hline t-Test: Two-Sample Assuming Unequal Variances & & \\
\hline & $H U @ 80 k v$ & $H U ~ @ 100 k v$ \\
\hline Mean & 643.88 & 387.34 \\
\hline Variance & 31691.57714 & 12539.20857 \\
\hline Observations & 50 & 50 \\
\hline Hypothesized Mean Difference & 0 & \\
\hline df & 83 & \\
\hline t Stat & 8.625370626 & \\
\hline $\mathrm{P}(\mathrm{T}<=\mathrm{t})$ one-tail & $1.83502 \mathrm{E}-13$ & \\
\hline $\mathrm{t}$ Critical one-tail & 1.663420175 & \\
\hline $\mathrm{P}(\mathrm{T}<=\mathrm{t})$ two-tail & $3.67004 \mathrm{E}-13$ & \\
\hline $\mathrm{t}$ Critical two-tail & 1.98895978 & \\
\hline Statistical sig diff $(\mathrm{p}<0.01)$ & & \\
\hline$(\mathrm{t}(83)=-8.6, \mathrm{p}<0.01$ & & \\
\hline
\end{tabular}

$$
\begin{gathered}
\mathrm{H}_{0}: \mu_{\text {Contrast enhancement } 80 \mathrm{kv}}=\mu_{\text {Contrast enhancement } 100 \mathrm{kv}} \\
\mathrm{H}_{\mathrm{A}}: \mu_{\text {Contrast enhancement 80v }}>\mu_{\text {Contrast enhancement } 100 \mathrm{kv}}
\end{gathered}
$$

$\boldsymbol{H}_{a}$ : The alternative hypothesis validates that contrast enhancement from low dose CT pulmonary angiogram is greater than that from the $100 \mathrm{kV}$ protocol. The low-dose considerably improved the contrast enhancement, and this increases diagnostic confidence and decreases suboptimal examinations. 
Table 6 shows radiologist overall image quality 3-point scale rating

\begin{tabular}{|c|c|}
\hline \multicolumn{2}{|c|}{ Radiologist 1 Overall image quality rating } \\
\hline Low dose 80kV protocol & $100 \mathrm{kV}$ standard imaging protocol \\
\hline Excellent (score 1), $n=34(68 \%)$ & Excellent (score 1), $n=35(70 \%)$ \\
\hline Good (score 2$), n=14(28 \%)$ & Good (score 2$), n=9(18 \%)$ \\
\hline $\begin{array}{l}\text { Suboptimal image quality (score } 3), \mathrm{n}=2(4 \%) \\
\text { *One case has Motion artefact. } \\
\text { * Other with reduced opacification of contrast on peripheral arteries. }\end{array}$ & $\begin{array}{l}\text { Suboptimal image quality (score } 3) \mathrm{n}=6(12 \%) \text {, } \\
* \text { five cases of low contrast enhancement } \\
* \text { One case of motion artefact. }\end{array}$ \\
\hline \multicolumn{2}{|c|}{ Radiologist 2 Overall image quality rating } \\
\hline Low dose $80 \mathrm{kV}$ protocol & $100 \mathrm{kV}$ standard imaging protocol \\
\hline Excellent (score 1), $n=29(58 \%)$ & Excellent (score 1), $n=27(54 \%)$ \\
\hline Good (score 2$), n=19(38 \%)$ & Good (score 2$), n=17(34 \%)$ \\
\hline $\begin{array}{l}\text { Suboptimal image quality (score } 3), \mathrm{n}=2(4 \%) \\
\text { *One case has Motion artefact. } \\
\text { * reduced opacification of contrast on peripheral arteries. }\end{array}$ & $\begin{array}{l}\text { Suboptimal image quality (score } 3) \mathrm{n}=6(12 \%) \text {, } \\
*_{\text {four cases of low contrast enhancement }} \\
* \text { One case of motion artefact. }\end{array}$ \\
\hline
\end{tabular}

\section{Pearson's Chi-squared test}

$$
\begin{aligned}
& \mathrm{H}_{0}: \mu_{\text {diagnostic confidence } 80 \mathrm{kv}} \neq \mu_{\text {diagnostic confidence } 100 \mathrm{kv}} \\
& \mathrm{H}_{\mathrm{A}}: \mu_{\text {diagnostic confidence } 80 \mathrm{kv}}=\mu_{\text {diagnostic confidence } 100 \mathrm{kv}}
\end{aligned}
$$

- Radiologist one: We have $\chi^{2}=3.10, \mathrm{df}=2$, $\mathrm{p}$-value $=0.212$.

- Radiologist two: We have $\chi^{2}=2.182, \mathrm{df}=2$, $\mathrm{p}$-value $=0.335$

The $\mathrm{p}$-value $>0.05$. We have sufficient evidence to accept the alternative hypothesis, which indicates that diagnostic confidence and image quality of $80 \mathrm{kV}$ are equal to that of the $100 \mathrm{kV}$ standard protocol.

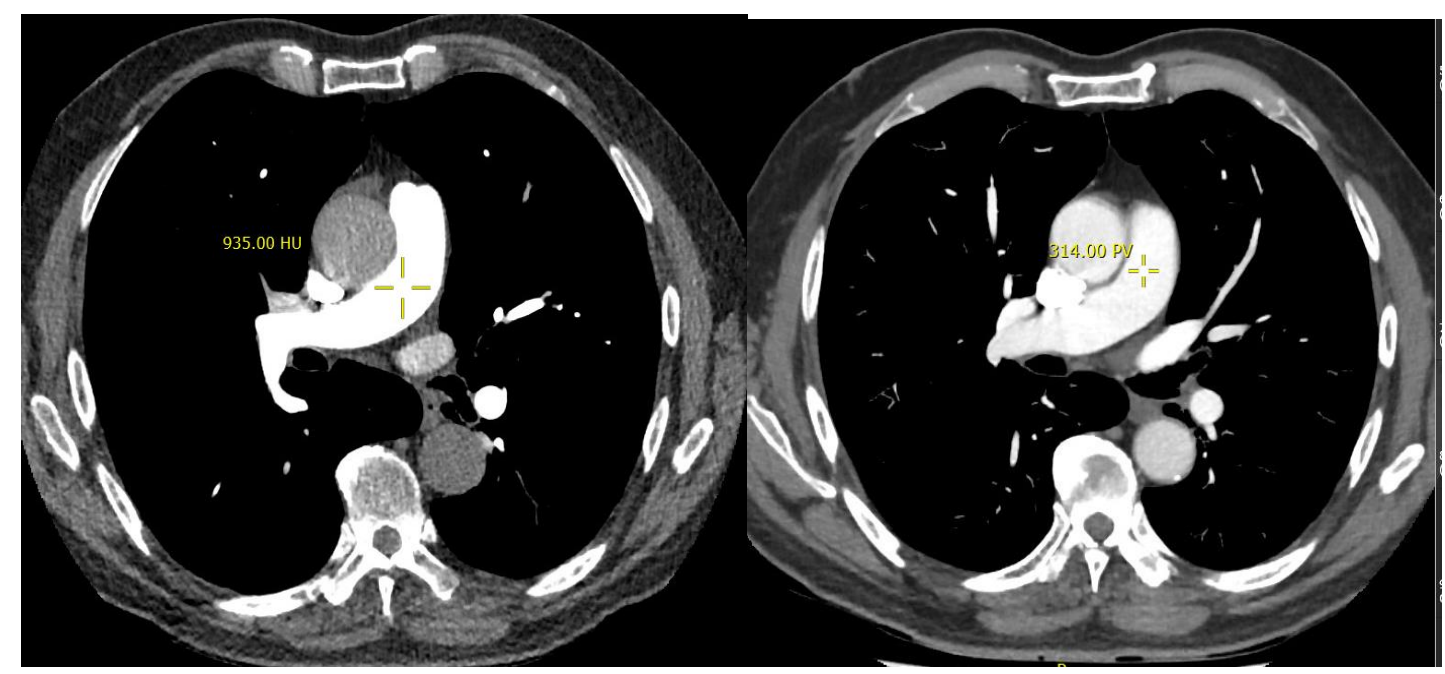

a

$\mathrm{b}$

Figure 3 (a) The mediastinal axial image with standard $100 \mathrm{kV}$ protocol; (b) Mediastinal axial image obtained using $80 \mathrm{kVp}$ protocol, which is a $75 \%$ reduction in radiation dose. Images acquired ten months apart. 


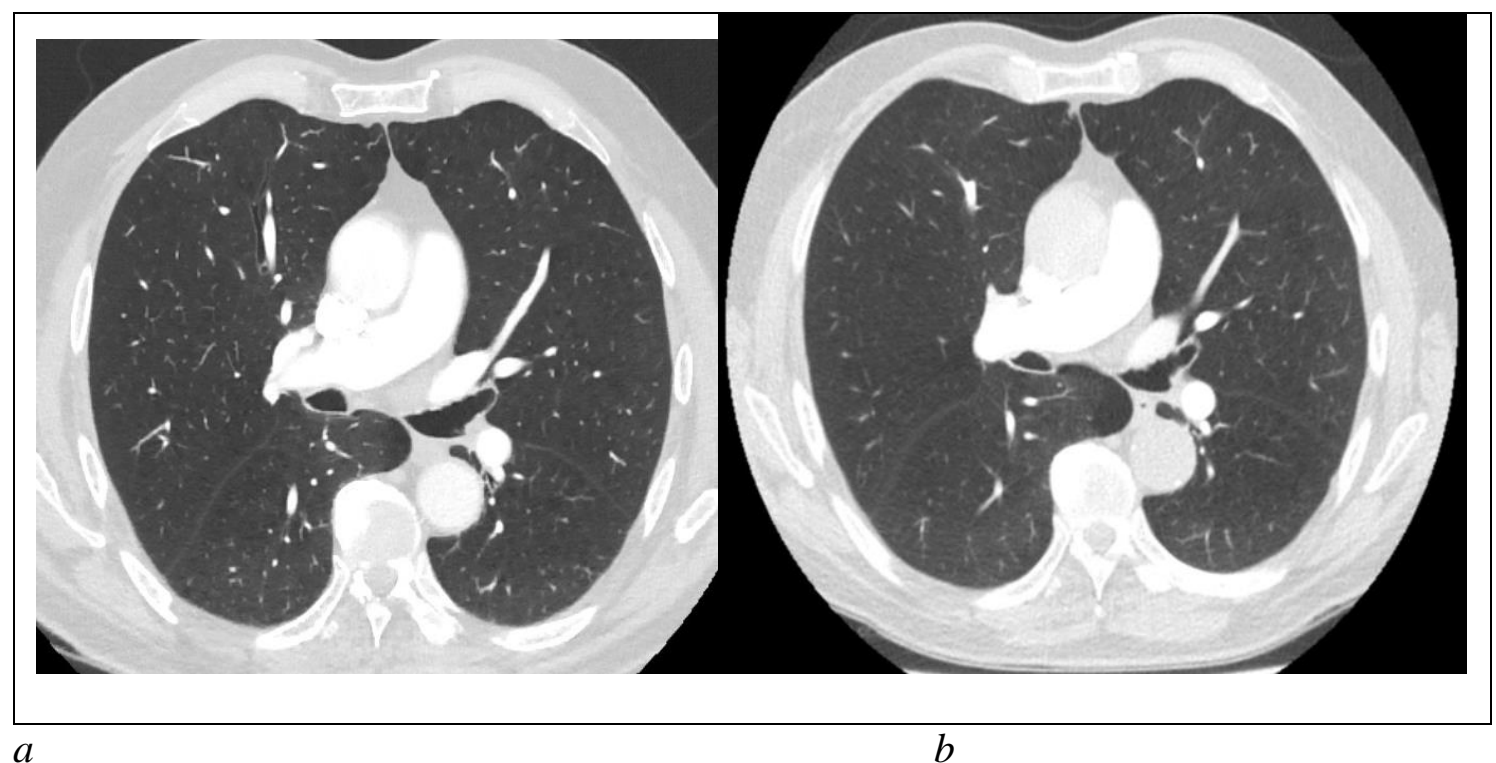

Figure 4 shows the lung window of the above images with no significant variance within the image quality: (a) lung window axial image obtained with the standard $100 \mathrm{kV}$ protocol and (b) lung window axial image obtained at the new low dose CT pulmonary angiogram protocol of $80 \mathrm{kV}$.

\section{DISCUSSION}

CTPA is an ideal assessment for PE imaging in many clinical situations. It offers accurate diagnostic specificity and sensitivity but does come at the cost of high radiation dose. Appropriate radiation dose reduction techniques are required without damaging image quality, as a significant drop in radiation dose may result in weakened image quality and consequently missed PE diagnoses.

We have found with the current research that an $80 \mathrm{kV}$ CTPA protocol can implement clinically which good imaging quality and low image noise. We have also found that radiation dose was reduced with the use of $80 \mathrm{kV}$, lower standard deviation, and enhanced image reconstructions algorithm. The low dose protocol achieved the image quality that was objectively similar to that obtained with a standard 100kV CTPA protocol. The low dose of CTPA generated a quality image consistent with the criteria set out in the European Union Quality Criteria For Computed Tomography Working Document ${ }^{7}$. Image quality criteria include clear visualization of structures, sharp visualization of pulmonary arteries, lung parenchyma, and pulmonary fissures. Clear visualization of a large, medium, and smallsized bronchi, as well as visually sharp visualisation of the border between the pleura and the thoracic wall ${ }^{7}$. Within the lung window, radiologists assessed the dominant pattern and distribution of the alternative pathologies.

Regarding spatial resolution, image noise, and contrast resolution, most radiologists revealed that image quality was acceptable and accurate in diagnosing or excluding PE with lower tube voltages. With the new $80 \mathrm{kV}$ protocol, image quality was maintained and rated either "excellent" or "good" in most cases. In our study, two patients out of 50 had suboptimal or mildly suboptimal CTPAs caused by reduced contrast opacification in sub-segmental arteries and motion artefact compared to 6 patients in the standard CTPA protocol group

The average radiation dose was significantly lower with the $80 \mathrm{kV}$ protocol than the $100 \mathrm{kV}$ protocol, 1.005 and $3.03 \mathrm{mSv}$, respectively. This is the lowest CTPA radiation dose available in Canberra's imaging departments and, most likely, Australia. These findings are comparable to the SzucsFarkas et al. (2008) study, which achieved a $40 \%$ radiation dose reduction even though our study's radiation dose saving is considerably higher than the above study ${ }^{8}$. 
Radiation dose is the main accomplishment of this study as high radiation exposure to patients is associated with elevated lifetime cancer risks. This study has also demonstrated that one of the limitations of previous studies, increased imaging noise, can be offset but utilizing a low standard deviation for the tube voltage and improved reconstruction algorithms. Although the studies found a mild increase in the image noise among larger patients on the lung window, this was also significantly impacted diagnostic confidence.

We were also able to retain the image's quality and reduce image noise through the use of the reconstruction algorithm FC5 and tube voltage standard deviation level 8 and the image reconstruction process AID 3D strong. The protocol also incorporated tube current modulation to track the fluctuating patient anatomy.

Other approaches to reduce radiation dose exists ${ }^{9,10}$. The most prominent involving reduced mAs. The disadvantages of utilizing a fixed or reduced mAs are the inability to offer a precise exposure for variable patient sizes unless an exposure chart is used, which is impractical for a busy imaging department. Thus, as with this study, an alternative technique is to reduce the $\mathrm{kV}$ with the current modulation; overall, this decreases radiation exposure in lower attenuation parts and provides adequate image quality.

Enhanced pulmonary arterial tree enhancement is also another significant advantage of this protocol. The study found considerable improvement in the contrast enhancement within the pulmonary arterial tree, which decreased the possibility of nondiagnostic scans. Several studies also reported similar findings ${ }^{11,12}$.

Contrast enhancement can be attributed to low tube voltage bringing the photon energy near the iodine K-edge. In turn, it increases the pulmonary arteries' contrast enhancement. Therefore detecting the PE filling defects may be easier to identify. Furthermore, this improved enhancement will allow clinicians, particularly in the emergency setting, to diagnose PE quickly, decreasing the time most patients spend in the ED. Moreover, low voltage protocol may also be advantageous, particularly to patients with a low glomerular filtration as well as those with restricted intravenous cannula access who may profit from decreased quantity of contrast agent, but this may be an avenue for future investigation.

\section{LIMITATIONS}

Several limitations are noted. One of the most significant limitations is that assessing radiologists may be biased either for or against a protocol based on imaging parameters visible on presented images. Another rule may be encountered from the radiologists' preference bias; they may prefer the brighter low dose images and assess them as better. Finally, the sample size is small; the test sample size of 50 patients can restrict the findings' generalization.

\section{CONCLUSION}

A low-dose CTPA protocol demonstrated a significant decreased in the radiation dose and simultaneous increased pulmonary artery contrast enhancement without compromising the diagnostic confidence or image quality.

\section{ACKNOWLEDGEMENTS}

The authors would like to acknowledge $\mathrm{Dr}$ Shahroz Khan, Dr Harith Al-Rawi, Dr Saidul Ansary, Dr John Connors and Hayley Scriven for their assistance in providing their low-dose CT pulmonary angiogram expertise. This doctoral research program is supported by the Australian Government Research Training Program (RTP).

\section{DISCLOSURE OF CONFLICT OF INTEREST}

The researchers claimed that existed no conflict of interest.

\section{REFERENCES}

1. Carroll BJ, Beyer SE, Mehegan T, Dicks A, Pribish A, Locke A, et al. Changes in Care for Acute Pulmonary Embolism with a Multidisciplinary Pulmonary Embolism Response Team: PE Response Team. The American Journal of Medicine. 2020. 
2. Ishaaya E, Tapson VF. Advances in the diagnosis of acute pulmonary embolism. F1000Research. 2020;9.

3. Aissaoui N, Konstantinides S, Meyer G. What's new in severe pulmonary embolism? Intensive care medicine. 2019;45(1):75-7.

4. Stulz P, Schliipfer R, Feer R, Habicht J, Griidel E. Decision making in the surgical treatment of massive pulmonary embolism. shock. 1994;19(15):79.

5. Romans L. Computed Tomography for Technologists: A comprehensive text: Lippincott Williams \& Wilkins; 2018.

6. Wittram C, Maher MM, Halpern EF, Shepard J-AO. Attenuation of acute and chronic pulmonary emboli. Radiology. 2005;235(3):1050-4.

7. Bongartz G, Golding S, Jurik, A, et al. European Union Quality Creteria Computed tomography. European Union website Established by the European Commission;s Study Group on Development of Quality Criteria for CT scan. 1998.

8. Szucs-Farkas Z, Kurmann L, Strautz T, Patak MA, Vock P, Schindera ST. Patient exposure and image quality of low-dose pulmonary computed tomography angiography: comparison of 100-and 80$\mathrm{kVp}$ protocols. Investigative radiology. 2008;43(12):871-6.

9. Rajiah P, Ciancibello L, Novak R, Sposato J, Landeras L, Gilkeson R. Ultralow dose contrast CT pulmonary angiography in oncology patients using a high-pitch helical dual-source technology. Diagn Interv Radiol. 2019;25(3):195-203.

10. Rusandu A, Ødegård A, Engh G, Olerud HM. The use of $80 \mathrm{kV}$ versus $100 \mathrm{kV}$ in pulmonary CT angiography: an evaluation of the impact on radiation dose and image quality on two CT scanners. Radiography. 2019;25(1):58-64.

11. Aldosari S, Sun Z. A Systematic Review of Double Low-dose CT Pulmonary Angiography in Pulmonary Embolism. Current Medical Imaging Reviews. 2019;15(5):453-60.

12. Mustafa K, Kayan M, Cetinkaya G, Turkoglu S, Kayan F. Investigating the use and optimization of low dose KV and contrast media in CT Pulmonary angiography examination. Iranian Journal of Radiology. 2018;15(3). 